\section{Pore performance}

Transport vesicles continuously shunt molecules around the cell, ensuring delivery to the correct intracellular destination. Essential to this process are the SNARE proteins, which mediate docking and attachment of vesicles with their target membrane. But how, once a vesicle contacts the target membrane, are the repulsive forces between the membranes overcome to allow fusion? Scientists remain divided on this point. Whereas some believe that SNAREs, by bringing membranes into close proximity, are sufficient for this step, others propose a fusion pore model, in which a proteineous channel forms. Now, reporting in Nature, Peters and colleagues reveal the identity of such a channel, and it turns out to be a familiar one.

Calcium and calmodulin can trigger the final step of fusion, but their downstream targets were not known. So, to identify calmodulin targets, Peters and colleagues used label-transfer analysis to isolate nearby factors on vacuolar membranes. Intriguingly, when they then purified these factors, mass spectrometry identified them as components of the $\mathrm{V}_{0}$ integral membrane sector of V-ATPase - a membrane complex that functions as a proton pump. The relevant calmodulin-binding partner was then found to be the $\mathrm{V}_{0}$ proteolipid.

So can these proteolipids trigger fusion? To test this, the authors monitored their ability to trigger choline release in reconstituted liposomes. And they found, consistent with the channel model, that they triggered release in a calcium/calmodulin-dependent manner.

Membrane docking requires formation of an electrochemical membrane potential, which is mainly generated by the V-ATPase. But if $\mathrm{V}_{0}$ is important for fusion itself then it should have a direct role in fusion, independent of proton pump activity. The authors confirmed this, showing that fusion could occur once they had knocked out proton pump activity.

So where do SNAREs come in? By inhibiting different stages of fusion, the authors showed that trans-SNARE pairing mediates channel formation, but not maintenance. One possibility, then, is that SNAREs concentrate $V_{0}$ sectors at the site of close membrane contact.

What remains to be seen is how, once formed, these channels allow bilayer mixing. The authors' bets are hedged on a model in which channels expand radially to form an aqueous pore but, as is often the case, more specific analytical tools are needed before we can dig any deeper.

6) References and links

ORIGINAL RESEARCH PAPER Peters, C. et al. Trans-complex formation by proteolipid channels in the terminal phase of membrane fusion. Nature 1 February (2001)

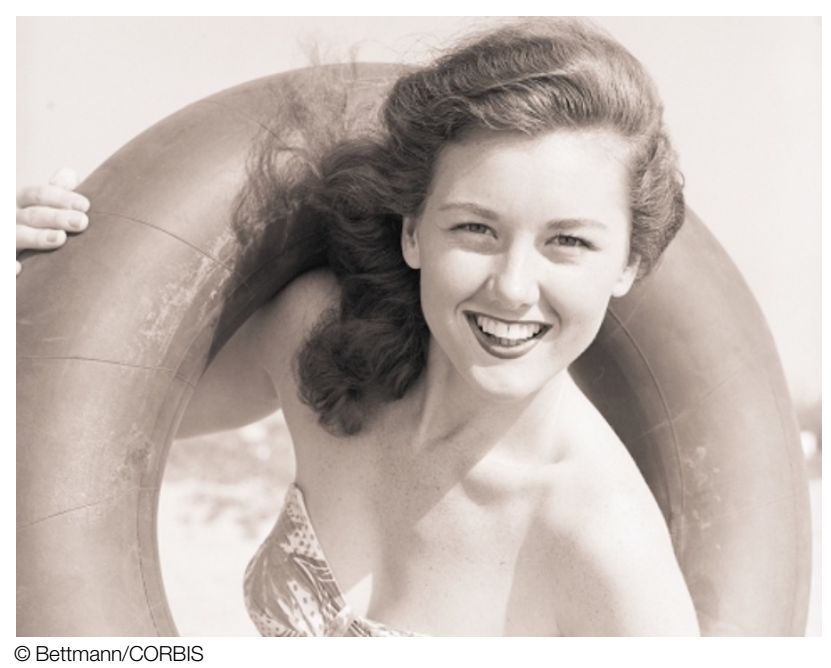

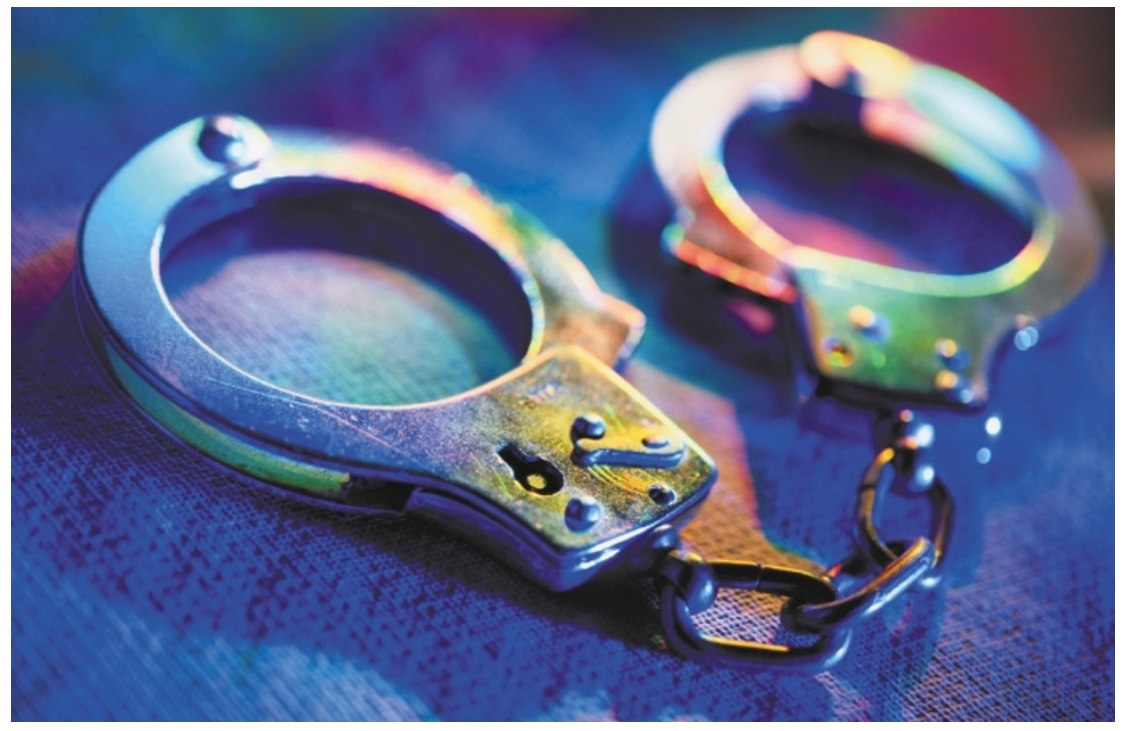

DNA REPAIR

\title{
Motif with a motive
}

Scientists, like policemen, often have to work backwards - start with the motive to track down the culprit. Reporting in Nature,

Downs et al. describe an example of such molecular detective work. They have linked phosphorylation of the Ser-Gln-Glu (SQE) motif in the yeast core histone $\mathrm{H} 2 \mathrm{~A}$ to alterations in chromatin alterations that might facilitate the repair of damaged DNA.

The SQE motif is phosphorylated in vitro by members of the phosphatidylinositol-3-OH kinaserelated kinase (PIKK) family. These include human ATM and ATR, and their Saccharomyces cerevisiae homologues Meclp and Tellp, all of which are central in eukaryotic responses to DNA damage.

Because the carboxyl terminus of histone H2A contains an SQE motif, Downs et al. wondered whether phosphorylation of this sequence might be involved in signalling DNA damage. They generated a strain in which the SQE motif was deleted, and found that it was hypersensitive to chemicals that induce DNA damage, such as methyl methane-sulphonate (MMS). When S, Q and E were mutated individually, the phenotypes correlated with the relative importance of each residue in defining an optimal PIKKrecognition motif. So could the hypersensitivity to MMS reflect loss of recognition by a PIKK? And if so, which one?

Strains with mutations in the
MEC1 gene could not phosphorylate the SQE motif of $\mathrm{H} 2 \mathrm{~A}$ in the presence of MMS, indicating that $\mathrm{H} 2 \mathrm{~A}$ might be a direct target for Meclp. Moreover, although phosphorylation of the SQE motif had no effect on Meclpdependent transcriptional or cellcycle responses to DNA damage, it did seem to be necessary for Meclp-dependent repair of doublestranded DNA breaks.

Given that $\mathrm{H} 2 \mathrm{~A}$ is a histone protein, one way of facilitating repair would be to alter the structure of chromatin around the broken DNA. This would allow the repair machinery easy access to the lesion. Consistent with this idea, Downs et al. found that in a mutant strain that mimicked the effect of phosphorylated SQE, the regions between individual nucleosomes were more sensitive to digestion by micrococcal nuclease - that is, compaction of the chromatin had been decreased.

The carboxyl terminus of $\mathrm{H} 2 \mathrm{~A}$ sits at the part of the nucleosome where DNA enters and exits, so it's ideally placed to influence the higher order structure of chromatin. How phosphorylation of the H2A carboxyl terminus leads to this effect is just one of the next lines of enquiry that the authors will be following up.

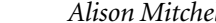

(2) References and links ORIGINAL RESEARCH PAPER Downs, J. A., Lowndes, N. F. \& Jackson, S. P. A role for Saccharomyces cerevisiae histone H2A in DNA repair. Nature 408, 1001-1004 (2000) 